Review

\title{
Role of TERRA in the Regulation of Telomere Length
}

\author{
Caiqin Wang, Li Zhao, Shiming Lu ${ }^{凶}$ \\ Key Laboratory of Reproductive Genetics (Zhejiang University), Ministry of Education, China, Women's Hospital, School of Medicine, \\ Zhejiang University, Xueshi Road 1\#, Hangzhou 310006, China
}

$\square$ Corresponding author: Shiming Lu, Key Laboratory of Reproductive Genetics (Zhejiang University), Ministry of Education, China, Women's Hospital, School of Medicine, Zhejiang University, Xueshi Road 1\#, Hangzhou 310006, China; E-mail: lusm@zju.edu.cn

(c) 2015 Ivyspring International Publisher. Reproduction is permitted for personal, noncommercial use, provided that the article is in whole, unmodified, and properly cited. See http://ivyspring.com/terms for terms and conditions.

Received: 2014.09.11; Accepted: 2014.12.25; Published: 2015.02.05

\begin{abstract}
Telomere dysfunction is closely associated with human diseases such as cancer and ageing. Inappropriate changes in telomere length and/or structure result in telomere dysfunction. Telomeres have been considered to be transcriptionally silent, but it was recently demonstrated that mammalian telomeres are transcribed into telomeric repeat-containing RNA (TERRA). TERRA, a long non-coding RNA, participates in the regulation of telomere length, telomerase activity and heterochromatinization. The correct regulation of telomere length may be crucial to telomeric homeostasis and functions. Here, we summarize recent advances in our understanding of the crucial role of TERRA in the maintenance of telomere length, with focus on the variety of mechanisms by which TERRA is involved in the regulation of telomere length. This review aims to enable further understanding of how TERRA-targeted drugs can target telomere-related diseases.
\end{abstract}

Key words: TERRA, telomere length, telomerase, HDR, ALT, protection of chromosome end

\section{Introduction}

Telomeres were identified in the 1930s and are nucleoprotein structures that protect the ends of chromosomes from being recognized and processed as DNA double-strand breaks (DSBs) and from degradation [1]. Human telomeres extend from 9-15 Kb, but can be as long as $100 \mathrm{~Kb}$ in rodents [1, 2]. Human and mouse telomeres are composed of long tracts of double-stranded G rich TTAGGG repeats and a telomere-specific protein complex, shelterin. The shelterin complex consists of six protein subunits: telomeric repeat binding factor-1 (TRF1), TRF2, repressor/activator protein-1 (RAP1), TRF1-interacting protein-2 (TIN2), TINT1/PIP1/PTOP 1 (TPP1) and protection of telomeres-1 (POT1), and regulates the maintenance of telomere length and protects natural chromosome ends from being recognized as damaged DNA [1, 3-5]. Single-stranded repeat structures created by $5^{\prime}$ to 3 ' exonucleases and termed G-overhangs protrude from the $3^{\prime}$ end of telomeres [6]. The invasion of the $3^{\prime}$ telomeric overhang into the duplex telomeric array forms a T-loop, and this process is promoted by TRF2. T-loops generated from the 3' G-overhang provide a protective cap that can protect against DNA damage induced by the DNA damage response (DDR) machinery [7].

Telomeres shorten following each cell division via a phenomenon termed the end-replication problem [8] and exonuclease-mediated processing [9]. When telomeres become shortened below a critical length, they can lose their protective function. To date, two mechanisms have been identified that prevent telomere shortening: firstly, telomere elongation by the ribonucleoprotein enzyme telomerase, telomerase adds telomeric repeats to chromosome ends and has a strong preference for short telomeres [10], and secondly, the telomere maintenance mechanism (TMM), which depends on homology-directed repair (HDR) to elongate telomeres and is also termed alternative lengthening of telomeres (ALT). ALT occurs in approximately $10-15 \%$ of human cancer cells [11-13], in which telomere elongation occurs in ALT-related PML bodies (APBs), a specific class of promyelocytic 
leukemia (PML) nuclear bodies that includes PML protein, telomeric DNA, telomere-specific proteins, and recombination and repair proteins [14]. Telomeric material is recruited to PML bodies to initiate recombination. In ALT cells, the presence of APBs plays a positive role in telomere maintenance; furthermore, PML bodies can modify telomere-associated chromatin and improve the stability of telomeres [15]. The role of APBs in telomere length maintenance remains unclear, although it has been suggested to be the site of telomere recombination, so we will not further discuss this problem here.

For many years, telomeres have been considered to be transcriptionally silent; in 2007, it was demonstrated that mammalian telomeres are indeed transcribed into telomeric repeat-containing RNA (TERRA) [16]. In a similar manner to long noncoding RNAs (lncRNAs), TERRA participates in the fine regulation of cell biology, which opens a new field in the understanding of telomeric functions and related diseases. TERRA molecules localize to telomeres and regulate telomerase activity, telomere length and associated heterochromatinization. Changes in the expression level of TERRA are associated with altered telomeric length and promote genome instability and cellular senescence. In this review, we discuss the present knowledge of TERRA biogenesis, structure and functional regulation, and particularly focuses on the various mechanisms that regulate telomere length.

\section{Biogenesis, structure and functional reg- ulation of TERRA}

Telomeric repeat-containing RNA (TERRA) is transcribed from several subtelomeric loci located close to chromosome ends, is composed of varying sizes of UUAGGG repeat sequences and ranges in size from $100 \mathrm{bp}$ to $9 \mathrm{~Kb}$ in mammals [16]. TERRA is expressed in most mammalian tissues. Approximately $7 \%$ of human TERRA molecules are polyadenylated [17] whereas most or all yeast TERRA molecules carry a poly(A) tail [18], and all human and most yeast TERRA $5^{\prime}$ ends contain a 7-methylguanosine $\left(\mathrm{m}^{7} \mathrm{G}\right)$ cap structure [19]; these features increase the stability of TERRA molecules. Furthermore, TERRA forms stable G-quadruplexes in vitro and in vivo [20-26], and there is direct evidence that TERRA binds to the telomeric protein TRF2 by forming an intramolecular G-quadruplex structure [25]; additional characterization of this structure may help to further understand the molecular interactions between nucleic acids and proteins at chromosome ends and design telomere-targeting loci for therapy in the future. TERRA is mainly transcribed by RNA polymerase II (RNAPII); this process has been reported to be regulated by telomeric heterochromatin and is promoted human TRF1; silencing of TRF1 using small interfering RNA (siRNA) decreased the expression of TERRA by two-fold in murine $\mathrm{C} 2 \mathrm{C} 12$ cells [27]. In yeast, the 5'-3' exonuclease, Rat1, negatively regulates the levels of TERRA via a mechanism that is mediated in part by the Saccharomyces cerevisiae repressor-activator protein 1, Rap1, and its interacting proteins, Rif1 and Rif2 [18, 28]. TRF4, an alternative poly(A) polymerase that is often associated with exosomal-mediated degradation, also plays a minor role in the degradation of TERRA, but only in Rat1-deficient cells. On the other hand, overexpression of RNAse $\mathrm{H}$, which degrades RNA in RNA-DNA hybrid molecules, reduced the expression of TERRA in budding yeast.

TERRA participates in multiple regulatory functions, such as telomerase activity [29-32], heterochromatinization of telomeres [33] and development, such as cellular differentiation [27, 34-36]. TERRA has been confirmed to associate with telomeres via tethering in a DNA-RNA hybrid formation $[18,37]$ or via interaction of RNA-binding proteins with telomeres [38]. For example, members of the heterogeneous nuclear ribonucleoprotein (hnRNP) family can assist in the binding of TERRA to telomeres and influence the levels of TERRA by regulating the stability of the transcripts [39]. Recently, systematic, comprehensive, quantitative proteomic analysis of proteins that interact with non-coding repetitive RNA led to the identification of novel interactors that play a role in TERRA homeostasis and its association with telomeres. This work indicated that the telomere binding proteins, TRF1, and the component of the Nua4 histone acetyltransferase complex, MORF4L2, may be required for the transcription or transcriptional stability of TERRA and telomere binding [40]. Further discovery of additional TERRA-associated proteins may help to more precisely characterize the function of TERRA.

\section{TERRA promotes telomere shortening}

For several years, research interest in TERRA mainly focused on yeast cells, human tumor cells and stem cells. In Saccharomyces cerevisiae, Rat1p, a yeast $5^{\prime}$ to $3^{\prime}$ RNA exonuclease, degrades TERRA and promotes telomere elongation; when Rat1p is mutated, TERRA accumulates and forms a DNA/RNA hybrid that inhibits telomerase activity at telomeres and results in telomere shortening [18]. Furthermore, TERRA interacts with the Exo1-inhibiting Ku70/80 dimer that promotes Exo1-dependent resection at chromosome ends during telomere shortening [41]. In the absence of a telomere maintenance mechanism, it has been reported that increased telomere transcription results in telomere shorting as a result of DNA replication-dependent loss of telomere tracts [42]. The 
THO complex is a multi-protein complex that promotes coupling between transcription and mRNA processing [43]. In S. cerevisiae, the minimal THO complex, composed of Tho2, Hpr1, Mft1 and Thp2, plays an important role in RNA maturation. Telomere shortening occurs when Thp2, a subunit of the THO complex, is deleted. In thp2-deleted yeast cells, the maturation of TERRA is prevented, and nascent TERRA entangles around the DNA template, interfering with chromosome end cap formation and protection from the telomere end-trimming enzyme Exo1 that induces telomere shortening [44]. It has also been demonstrated TERRA forms tRNA-DNA hybrids or R-loops at chromosome ends [44]. Bettina and colleagues discovered that the accumulation of RNA-DNA hybrids at telomeres led to rapid senescence and telomere loss in the absence of telomerase and HDR. In the absence of telomerase and presence of HDR, the increased accumulation of TERRA at shortened telomeres promotes telomere elongation via recombination processes, leading to maintenance of telomere length $[45,46]$. At shortened telomeres, TERRA is expressed and accumulates into nuclear foci that contain telomerase components, and these TERRA-telomerase clusters recruit telomerase to shortened telomeres from which TERRA originates during the early S phase [47]. During the late $S$ phase, telomerase preferentially elongates the shortest telomere [48] and the levels of TERRA decline at telomeres [49]. It has been reported that TERRA inhibits telomerase activity in vitro by binding to both the telomerase RNA (TR) moiety [27] and the telomerase reverse transcriptase (TERT) polypeptide independently of hTR [30]. These findings raised many questions regarding during which phases of the cell cycle TERRA regulates telomerase activity and how TERRA coordinates the recruitment of telomerase to telomeres and inhibits telomerase activity.

Mammalian subtelomere regions are packed into constitutive heterochromatin, which is characterized by high levels of histone 3 lysine 9 trimethylation (H3K9me3), histone 4 at Lys20 (H4K20) trimethylation and CpG methylation [50]. Decreases in the levels of these markers of heterochromatin suggest relative chromatin relaxation and are accompanied by telomere shortening [51]. The regulation of heterochromatinization by TERRA was initially reported in 2009 [33]. Over the following years, this function has been further clarified. In human immunodeficient lymphoblastoid and fibroblast cells, such as those resulting from patients with centromeric region instability or facial anomalies (ICF ) syndrome, subtelomeric regions are hypomethylated as a result of the mutation of DNA methyltransferase 3b (Dnmt3b), which performs de novo methylation of the genome during the early stages of mammalian embryonic development [52], and telomere length is abnormally reduced both in telomerase-positive and -negative cells. Additionally, TERRA is expressed at higher levels in ICF fibroblasts than control fibroblasts [53]. Furthermore, Deng et al. [54] identified multiple defects at the subtelomere regions in ICF cells, including a loss of CpG methylation, loss of TRF2 binding, and an absence of the heterochromatic histone marker H3K9me3. Additionally, the levels of the euchromatic histone modification $\mathrm{H} 3 \mathrm{~K} 4 \mathrm{me} 2$, which has been proven to regulate the transcription of TERRA by the mixed lineage leukemia (MLL) protein, a histone methyltransferase, are significantly increased in ICF cells [55]. It is speculated that TERRA facilitates heterochromatin formation via a negative feedback loop mechanism in normal cells, which is likely to be mediated by the recruitment of Dnmt3b and generation of CpG methylation within subtelomere regions. Thereafter, TERRA further stabilizes telomeric heterochromatin by physically interacting with Origin Recognition Complex (ORC), methyl CpG binding protein (MeCP2) and heterochromatin protein 1 (HP1). Due to the absence of $\mathrm{CpG}$ methylation at subtelomeres in DNMT3b-mutant ICF cells, TERRA cannot complete this negative feedback loop, resulting in an "open" chromatin state. Thus, induction of high levels of TERRA may promote telomere shortening in ICF cells by inhibiting telomerase activity, in conjunction with other mechanisms. Recently, using a model of induced pluripotent stem cells (iPSCs) from patients with type I ICF, it was demonstrated that high levels of TERRA do not restrict telomere lengthening in undifferentiated ICF-iPSCs due to upregulation of telomerase activity and the activation of the ALT pathway [36]. These results indicate that the ability of TERRA to regulate telomere length varies depending on the specific physiological conditions or developmental states.

Using human cell lines of various origins, Arnoult et al. found that telomere elongation consistently repressed the expression of TERRA by increasing the density of trimethylated $\mathrm{H} 3 \mathrm{~K} 9$ at telomeres and by heterochromatin protein HP1a [56]. Translocated in liposarcoma (TLS) protein, also termed FUS, (TLS/FUS) was initially identified as a human telomere-binding protein [57]. TLS binds to the double-stranded regions of G-quadruplex telomeric DNA and G-quadruplex TERRA, which in turn increases H4K20 trimethylation and leads to telomere shortening [58]. Furthermore, TERRA can recruit lysine-specific demethylase 1 (LSD1) to uncapped telomeres and trigger removal of telomeric 3' G overhangs by MRE11, one of the nucleases implicated in the processing of 3 'telomeric $G$ overhangs, and possi- 
bly other LSD1 substrates [59]; this may directly affect the ability of telomeres to protect linearized chromosome ends from being recognized as sites of DNA damage, as LSD1 and MRE11 participate in telomeric 3' $\mathrm{G}$ overhang removal during telomere end fusion.

In conclusion, TERRA participates in the regulation of telomere length via several pathways. Firstly, TERRA can inhibit telomerase activity to promote telomere shortening. Secondly, TERRA can promote Exo1-dependent resection at chromosome ends to initiate telomere shortening. TERRA also increases euchromatin formation or decreases heterochromatin formation to regulate telomere length. Additional functions of TERRA may be revealed in the future, and such discoveries will have a far-reaching impact on the study of telomere-associated diseases.

\section{TERRA regulates telomere length while protecting chromosome ends}

As discussed above in section "Introduction", telomeres become elongated via two mechanisms: telomerase adds DNA bases to telomere ends and by homology-directed repair (HDR). The telomere shelterin complex functions to regulate the maintenance of telomere length and can also protect natural chromosome ends from being recognized as damaged DNA. Shelterin is required to recruit telomerase to telomeres; in the absence of shelterin binding sites, telomerase cannot add telomeric DNA to the broken ends [60]. In human and Schizosaccharomyces pombe telomeres, the loss of TRF1 increases the recruitment of telomerase. Rap1 binds to the telomeres via TRF2 and plays a negative regulatory role on telomerase recruitment. The loss of POT1 from vertebrate telomeres results in telomere extension, which indicates a negative regulatory role for telomerase access [60]. Mammals possess unique DNA damage signaling and DNA repair pathways, in which the ATM and ATR kinase pathways are activated to initiate the repair of DNA nick damage by either the non-homologous end joining (NHEJ) or homology-directed repair (HDR) DNA repair pathways, allowing cells to continue to divide with an intact genome. However, these damage and repair events create several conflicting issues: firstly, activation of the ATM and ATR kinase pathways can lead to cell cycle arrest and induce cell death, on the other hand, the NHEJ pathway can produce chromosome end-to-end fusions whereas the HDR pathway can induce erroneous sequence exchanges. Interestingly, in mammals, this telomere end-protection problem is solved by the telomere shelterin complex and an altered structure (T-loop), which together ensure that all of the ATM, ATR, HDR and NHEJ pathways remain blocked. During this process, the shelterin component TRF2 represses the
ATM kinase signaling pathway and HDR by forming T-loops, and POT1 inhibits the ATR kinase pathway by preventing binding of replication protein A (RPA), a single-stranded DNA binding protein that activates the ATR kinase pathway, to the single-stranded telomeric DNA. On the other hand, during repair, although both TRF2 and POT1 can inhibit both HDR and NHEJ at telomeres, TRF2 plays a predominant role in inhibiting NHEJ before DNA replication starts; after DNA replication, both TRF2 and POT1 can inhibit the NHEJ pathway.

Recent studies showed that TERRA participates in telomere end protection; however, how does TERRA regulate telomere length during this process? The shelterin components TRF1 and TRF2 directly associate with TERRA in multiple cell types in vivo [33]. In vitro, T-loops were showed to contain TRF2 protein at the loop-tail junction and TRF2 promoted T-loop formation [7]. It has also been reported that the T-loops can be deleted by homologous recombination (HR), which results in the rapid shortening of telomeres. TRF2 is proposed to suppress T-loop HR by inhibiting branch migration and/or strand cleavage [61]. The amino-terminal define (GAR) domain of TRF2 has a high affinity for RNA; therefore, binding of TERRA to TRF2 could alleviate protection of the t-loop and induce t-loop HDR, which in turn would regulate the ability of TRF2 to condense DNA [62]. Indeed, it was reported that TERRA RNA depletion caused an increase in 53BP1 and $\gamma \mathrm{H} 2 \mathrm{AX}$-associated telomere foci [33], which belong to the family of DNA damage response proteins. In this manner, TERRA plays a positive role in the protection of telomere ends. This result appears to conflict with the report that TERRA might hinder t-loop formation [62], but the protection of telomere end might be a dual function of TERRA, which may need more refined regulatory mechanism. Using ChIP-seq, Deng et al. found that most human subtelomere regions contain a chromatin organizing factor (CTCF)- and cohesin-binding site, which is implicated in the regulation of TERRA transcription. When CTCF was knocked-down using siRNA in U2OS and HCT116 cells, the levels of endogenous TERRA reduced to a steady-state. Mechanistically, CTCF recruits RNAPII to subtelomere regions, and furthermore, CTCF and cohesin stabilize TRF bound to subtelomere regions to prevent telomere DNA damage signaling [63]. Additionally, the THO complex, via interplay with TERRA, regulates chromosome end processing activities and prevents interference with semi-conservative DNA replication [44]; in other words, the THO complex represses the formation of R-loops or TERRA-DNA hybrids at chromosome ends to prevent telomere shortening. In Saccharomyces cerevisiae, it has been 
proposed that the natural levels of TERRA-DNA hybrids prevent cellular senescence by promoting recombination-mediated telomere elongation events at critically shortened telomeres [64]. Subsequent studies proved that TERRA can promote telomere elongation in both telomerase negative- and telomerase-positive cells via HDR and telomerase-mediated elongation, respectively $[45,46]$. These findings imply that the levels of total TERRA must be strictly controlled during every phase of the cell cycle in order to avoid inappropriate changes in telomere length. Based on the findings of these recent data, we propose a model to illustrate the mechanism by which TERRA regulates telomere length by protecting chromosome ends in conjunction with TRF2 (Fig. 1).

The shelterin component POT1, a single-stranded DNA (ssDNA)-binding protein, plays a crucial role in telomere capping. POT1 and RPA coordinate to regulate telomere replication and capping. In spite of POT1 antagonizing the ability of RPA to bind telomeric ssDNA, this may not be sufficient to prevent RPA binding telomeric ssDNA. In order to investigate this apparently competitive process, Flynn

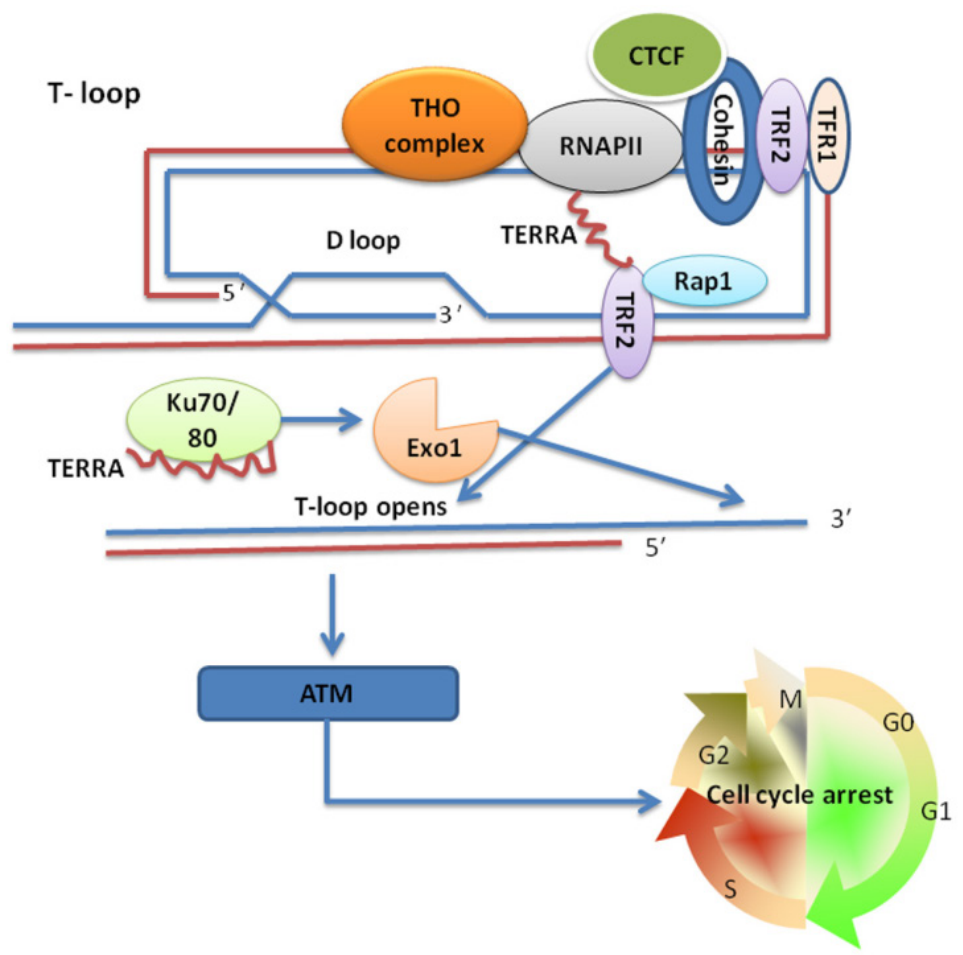

Fig. 1. TERRA regulation of telomere length for the protection of chromosome ends involving TRF2. CTCF recruits RNAPII to the CPG-island promoter and promotes TERRA transcription, CTCF and cohesin stabilization and TRF1/TRF2 binding to subtelomeres prevents telomere DNA damage signaling. The binding of TERRA to TRF2 hinders the formation of T-loops and activates the ATM kinase pathway. The THO complex enables the packaging of TERRA into RNPs and inhibits unscheduled TERRA expression, which could avoid the redundant removal of TERRA from the T-loop. TERRA interacts with the Exol-inhibiting Ku70/80 dimer and promotes Exol-dependent resection at chromosome ends to shorten the telomere. et al. identified that heterogeneous nuclear ribonucleoprotein A1 (hnRNPA1), which binds telomeres and participates in maintenance of telomere length [65,66], can remove RPA - but not POT1 - from telomeric ssDNA in vitro. On the basis of previous reports that hnRNPA1 binds TERRA [39], it was intriguing to explore if TERRA plays a role in hnRNPA1 displacing RPA from single-stranded telomeric DNA. Flynn et al. subsequently confirmed that TERRA can inhibit the ability of hnRNPA1 to displace RPA [67]. Due to the characteristic fluctuations in TERRA during cell cycle [49], the group proposed a model in which tethering of TERRA to hnRNPA1 contributes to the RPA-to-POT1 switch on telomeric ssDNA [68]. In early to mid $S$ phase, high levels of TERRA bind to hnRNPA1, which enables RPA to bind single-stranded telomeric DNA and release POT1 to enable telomere extension. During the late $S$ phase, TERRA levels decline and hnRNPA1 is released to displace RPA from single-stranded telomeric DNA. Between $G_{2}$ and $G_{1}$ phase, TERRA gradually re-accumulates, hnRNPA1 binds to TERRA and promotes binding of POT1 to telomeric ssDNA, ensuring POT1 functions to cap telomeres until the next $S$ phase. Thus, TERRA acts in combination with hnRNPA1 to regulate telomere replication and end protection.

Recently, Le and colleagues identified that DNA-dependent protein kinase catalytic subunit (DNA-PKcs) plays an important role the removal of TERRA from telomeres [69]. DNA-PKcs can promote phosphorylation of hnRNPA1, via a process reported to be activated by the hTR component of telomerase [70]. Le et al. demonstrated that inhibition of DNA-PKcs kinase activity or siRNA depletion of hnRNP A1 induced accumulation of TERRA at individual telomeres. Interestingly, as mentioned in section "TERRA promotes telomere shortening", TERRA can inhibit telomerase activity by binding to telomerase reverse transcriptase (TERT) and telomerase RNA moiety (TR). Therefore, we speculate that the dynamic balance of TERRA is a crucial factor which regulates telomere length in each phase of the cell cycle. For example, in early to mid $S$ phase, TERRA binds hnRNP A1 to participate in telomere replication and, during the same phase, is also necessary to inhibit telomerase activity to regulate telomere length. This appears to be conflicting, but might represent a more subtle regulatory process. Moreover, the regulation of telomerase activity is dependent on the cell cycle [71]. During the $S$ phase, telomerase 
activity is maximal, but following G2 to M, it decreases to a minimum. This activity gradually increases as the cells process through the G1/S phase [71]. It is fascinating to explore this regulation by TERRA and telomerase of the telomere replication and end protection during every phase of the cell cycle. Based on the figure from Flynn et al. 2012, we have attempted to illustrate this seemingly contradictory but subtle regulatory mechanism in Figure 2.

\section{Conclusions and Prospects}

As highlighted in this review, TERRA is a critical regulatory factor that controls telomeric length. TERRA can promote telomere shortening by inhibiting telomerase activity, promoting Exo1-dependent resections and both increasing the formation of euchromatin and decreasing heterochromatin formation. On the other hand, in the protection of chromosome ends, TERRA plays a negative role in maintenance of telomere length by binging to TRF2 and interacting with the Exo1-inhibiting Ku70/80 dimer. Indeed, TERRA participates in the regulation of various physiological processes, such as telomerase activity and heterochromatinization, and disruptions to these processes can affect development and directly or indirectly induce disease.

Excitingly, Reig-Viader and colleagues have

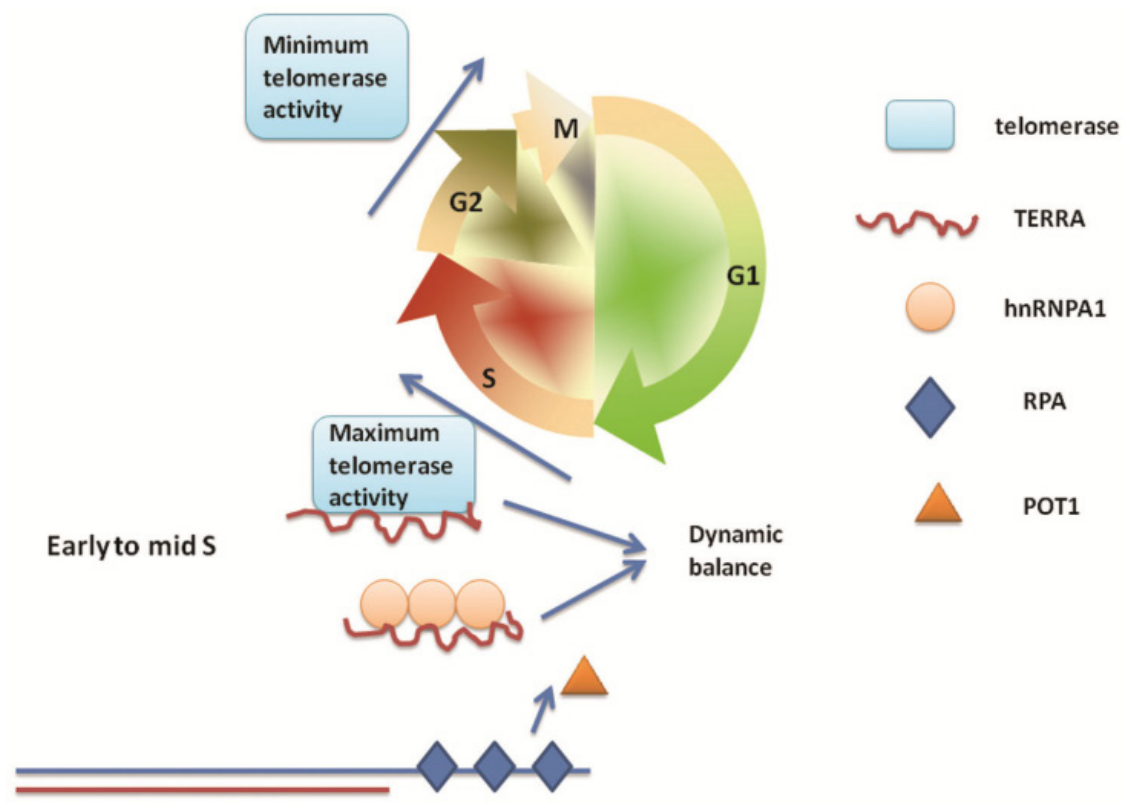

Fig. 2. Dynamic balance of TERRA sustains telomere length during telomere replication. TERRA acts as an on-switch for RPA-to-POT1 to regulate telomere replication and end protection. Changes in the level of TERRA are in accordance with changes in cell cycle-dependent telomerase activity. During the early to mid $S$ phase, high levels of TERRA bind to hnRNPA I, allowing RPA to bind ssTEL and releasing POT 1 to enable telomere extension; at the same time, TERRA binds to TERT or TERC to inhibit telomerase activity. The dynamic balance of TERRA is necessary to regulate these seemingly conflicted processes in order to maintain a normal telomere length. identified that TERRA is expressed during spermatogenesis and colocalizes with telomeres in mammalian meiocytes [72]. Reports that TERRA is expressed in fetal oocytes [73] indicate that further research on the role of TERRA may provide new advances in the field of reproduction. The ability of TERRA to regulate telomere length is also related to other human diseases, such as astrocytoma. In human astrocytoma cell lines, TERRA levels show down-regulation following tumor grades (from grade 4 to nonmalignant tissue), furthermore, TERRA expression is related to telomerase activity and telomere length [74]. It is expected that further exploration of the mechanisms by which TERRA regulates telomere length may provide new targets for the diagnosis and therapy of telomere-associated diseases.

\section{Abbreviations}

TERRA: telomeric repeat-containing RNA; DSBs: DNA double-strand breaks; TRF1: telomeric repeat binding factor-1; TRF2: telomeric repeat binding factor-2; RAP1: repressor/activator protein-1; TIN2: TRF1-interacting protein-2; POT1: protection of telomeres-1; DDR: DNA damage response; TMM: telomere maintenance mechanism; HDR: homology-directed repair; ALT: alternative lengthening of telomeres; PML: promyelocytic leukemia; lncRNAs: long noncoding RNAs; hnRNP: heterogeneous nuclear ribonucleoprotein; TR: telomerase RNA; TERT: telomerase reverse transcriptase; H3K9me3: histone 3 lysine 9 trimethylation; H4K20: histone 4 at Lys20; ICF: centromeric region instability or facial anomalies; Dnmt3b: DNA methyltransferase 3b; MLL: mixed lineage leukemia; ORC: Origin Recognition Complex; MeCP2: methyl CpG binding protein; HP1: heterochromatin protein 1; iPSCs: induced pluripotent stem cells; TLS: Translocated in liposarcoma; LSD1: lysine-specific demethylase 1; HDR: homology-directed repair; NHEJ: non-homologous end joining; CTCF: chromatin organizing factor; ssDNA: single-stranded DNA; hnRNPA1: heterogeneous nuclear ribonucleoprotein A1; DNA-PKcs: DNA-dependent protein kinase catalytic subunit. 


\section{Acknowledgements}

This work was supported by a grant from the Program for Key Innovative Research Team in Science \& Technology of Zhejiang Province, P. R. China [grant number 2011R50013].

\section{Competing Interests}

The authors have declared that no competing interest exists.

\section{References}

1. de Lange T. How telomeres solve the end-protection problem. Science. 2009; 326: 948-952.

2. O'Sullivan R, Karlseder J. Telomeres: protecting chromosomes against genome instability. Nat Rev Mol Cell Biol. 2010; 11: 171-181.

3. de Lange T. Shelterin: the protein complex that shapes and safeguards human telomeres. Genes Dev. 2005; 19: 2100-2110.

4. Palm W, de Lange T. How shelterin protects Mammalian telomeres. Annu. Rev. Genet. 2008; 42: 301-334.

5. Walker JR, Zhu XD. Post-translational modifications of TRF1 and TRF2 and their roles in telomere maintenance. Mech Ageing Dev. 2012; 133: 421-434.

6. Verdun RE, Karlseder J. Replication and protection of telomeres. Nature. 2007; 447: 924-931.

7. Griffith JD, Comeau L, Rosenfield S, Stansel RM, Bianchi A, Moss H, de Lange T. Mammalian telomeres end in a large duplex loop. Cell. 1999; 97: 503-514.

8. Watson JD. Origin of concatemeric T7 DNA. Nat New Biol. 1972; 239: 197-201.

9. Lingner J, Cooper JP, Cech TR. Telomerase and DNA end replication: no longer a lagging strand problem? Science. 1995; 269: 1533-1534.

10. Teixeira MT, Arneric M, Sperisen P, Lingner J. Telomere length homeostasis is achieved via a switch between telomerase-extendible and -nonextendible states. Cell. 2004; 117: 323-335.

11. Bryan TM, Englezou A, Dalla-Pozza L, Dunham MA, Reddel RR. Evidence for an alternative mechanism for maintaining telomere length in human tumors and tumor-derived cell lines. Nat Med. 1997; 3: 1271-1274.

12. Reddel RR. The role of senescence and immortalization in carcinogenesis. Carcinogenesis. 2000; 21:477-484.

13. Cesare AJ, Reddel RR. Alternative lengthening of telomeres: models, mechanisms and implications. Nat Rev Genet. 2010; 11: 319-330.

14. Yeager T R, Neumann AA, Englezou A, Huschtscha LI, Noble JR, Reddel RR. Telomerase-negative immortalized human cells contain a novel type of promyelocytic leukemia (PML) body. Cancer Res. 1999; 59: 4175-4179.

15. Draskovic I, Londono Vallejo A. Telomere recombination and alternative telomere lengthening mechanisms. Front Biosci (Landmark Ed). 2013; 18: 1-20.

16. Azzalin CM, Reichenbach $\mathrm{P}$, Khoriauli L, Giulotto E, Lingner J. Telomeric repeat containing RNA and RNA surveillance factors at mammalian chromosome ends. Science. 2007; 318:798-801.

17. Azzalin CM, Lingner J. Telomeres: the silence is broken. Cell Cycle. 2008; 7: 1161-1165.

18. Luke B, Panza A, Redon S, Iglesias N, Li Z, Lingner J. The Rat1p 5' to 3' exonuclease degrades telomeric repeat-containing RNA and promotes telomere elongation in Saccharomyces cerevisiae. Mol Cell. 2008; 32: 465-477.

19. Feuerhahn S, Iglesias N, Panza A, Porro A, Lingner J. TERRA biogenesis, turnover and implications for function. FEBS Lett. 2010; 584: 3812-3818.

20. Xu Y, Suzuki Y, Ito K, Komiyama M. Telomeric repeat-containing RNA structure in living cells. Proc Natl Acad Sci USA. 2010; 107: 14579-14584.

21. Collie GW, Haider SM, Neidle S. Parkinson GN. A crystallographic and modelling study of a human telomeric RNA (TERRA) quadruplex. Nucleic Acids Res. 2010; 38: 5569-5580.

22. Martadinata H, Heddi B, Lim KW, Phan AT. Structure of long human telomeric RNA (TERRA): G-quadruplexes formed by four and eight UUAGGG repeats are stable building blocks. Biochemistry. 2011; 50: 6455-6461.

23. Collie GW, Sparapani S, Parkinson GN, Neidle S. Structural basis of telomeric RNA quadruplex--acridine ligand recognition. J Am Chem Soc. 2011; 133: 2721-2728.

24. $\mathrm{Xu}, \mathrm{Y}, \mathrm{Komiyama} \mathrm{M}$. Structure, function and targeting of human telomere RNA. Methods. 2012; 57: 100-105.

25. Biffi G, Tannahill D, Balasubramanian S. An intramolecular G-quadruplex structure is required for binding of telomeric repeat-containing RNA to the telomeric protein TRF2. J Am Chem Soc. 2012; 134: 11974-11976.

26. Takahama K, Oyoshi T. Specific binding of modified RGG domain in TLS/FUS to G-quadruplex RNA: tyrosines in RGG domain recognize 2'-OH of the riboses of loops in G-quadruplex. J Am Chem Soc. 2013; 135: 18016-18019.

27. Schoeftner S, Blasco MA. Developmentally regulated transcription of mammalian telomeres by DNA-dependent RNA polymerase II. Nat Cell Biol. 2008; 10: $228-236$.

28. Iglesias N., Redon S., Pfeiffer V., et al. Subtelomeric repetitive elements determine TERRA regulation by Rap1/Rif and Rap1/Sir complexes in yeast. EMBO Rep 2011;12: 587-93.
29. Ng LJ, Cropley JE, Pickett HA, Reddel RR, Suter CM. Telomerase activity is associated with an increase in DNA methylation at the proximal subtelomere and a reduction in telomeric transcription. Nucleic Acids Res. 2009; 37: 1152-1159.

30. Redon S, Reichenbach P, Lingner J. (2010) The non-coding RNA TERRA is a natural ligand and direct inhibitor of human telomerase. Nucleic Acids Res. 2010; 38: 5797-5806.

31. Farnung BO, Brun CM, Arora R, Lorenzi LE, Azzalin CM. (2012) Telomerase efficiently elongates highly transcribing telomeres in human cancer cells. PLoS One. 2012; 7: e35714.

32. Redon S, Zemp I, Lingner J. A three-state model for the regulation of telomerase by TERRA and hnRNPA1. Nucleic Acids Res. 2013; 41: 9117-9128.

33. Deng Z, Norseen J, Wiedmer A, Riethman H, Lieberman PM. TERRA RNA binding to TRF2 facilitates heterochromatin formation and ORC recruitment at telomeres. Mol Cell. 2009; 35: 403-413.

34. Zhang LF, Ogawa Y, Ahn JY, Namekawa SH, Silva SS, Lee JT. Telomeric RNAs mark sex chromosomes in stem cells. Genetics. 2009; 182: 685-698.

35. Marion RM, Strati K, Li H, Tejera A, Schoeftner S, Ortega S, Serrano M, Blasco MA. Telomeres acquire embryonic stem cell characteristics in induced pluripotent stem cells. Cell Stem Cell. 2009; 4: 141-154.

36. Sagie S, Ellran E, Katzir H, Shaked R, Yehezkel S, Laevsky I, Ghanayim A, Geiger D, Tzukerman M, Selig S. Induced pluripotent stem cells as a model for telomeric abnormalities in ICF type I syndrome. Hum Mol Genet. 2014; 23: 3629-3640.

37. $\mathrm{Xu} Y$, Kimura T, Komiyama M. Human telomere RNA and DNA form an intermolecular G-quadruplex. Nucleic Acids SympSer (Oxf). 2008; 52: 169-170.

38. Dejardin J, Kingston RE. Purification of proteins associated with specific genomic loci. Cell. 2009; 136: 175-186.

39. López de Silanes I, Stagno d'Alcontres M, Blasco MA. TERRA transcripts are bound by a complex array of RNA-binding proteins. Nat Commun. 2010; 1: 33.

40. Scheibe M, Arnoult N, Kappei D, Buchholz F, Decottionies A, Butter F, Mann M. Quantitative interaction screen of telomeric repeat-containing RNA reveals novel TERRA regulators. Genome Res. 2013; 23: 2149-2157.

41. Pfeiffer $\mathrm{V}$, Lingner J. TERRA promotes telomere shortening through exonuclease 1-mediated resection of chromosome ends. PLoS Genet. 2012; 8: e1002747.

42. Maicher A, Kastner L, Dees M, Luke B. Deregulated telomere transcription causes replication-dependent telomere shortening and promotes cellular senescence. Nucleic Acids Res. 2012; 40: 6649-6659.

43. Rondon AG, Jimeno S, Aguilera A. The interface between transcription and mRNP export: from THO to THSC/TREX-2. Biochim Biophys Acta. 2010; 1799: 533-538

44. Pfeiffer V, Crittin J, Grolimund L, Lingner J. The THO complex component Thp2 counteracts telomeric R-loops and telomere shortening. EMBO J. 2013; 32: 2861-2871.

45. Yu TY, Kao YW, Lin JJ. Telomeric transcripts stimulate telomere recombination to suppress senescence in cells lacking telomerase. Proc Natl Acad Sci U S A. $2014 ; 111$ : 3377-3382.

46. Balk B, Dees M, Bender K, Luke B. The differential processing of telomeres in response to increased telomeric transcription and RNA-DNA hybrid accumulation. RNA Biol. 2014; 11: 95-100.

47. Cusanelli E, Romero CA. Chartrand P. Telomeric noncoding RNA TERRA is induced by telomere shortening to nucleate telomerase molecules at short telomeres. Mol Cell. 2013; 51: 780-791.

48. Teixeira MT, Arneric M, Sperisen P, Lingner J. Telomere length homeostasis is achieved via a switch between telomerase- extendible and -nonextendible states. Cell. 2004; 117: 323-335.

49. Porro A, Feuerhahn S, Reichenbach P, Lingner J. Molecular dissection of TERRA biogenesis unveils the presence of distinct and multiple regulatory pathways. Mol. Cell. Biol. 2010; 30: 4808-4817.

50. Blasco MA. The epigenetic regulation of mammalian telomeres. Nat Rev Genet. 2007; 8: 299-309.

51. Benetti R, García-Cao M, Blasco MA. Telomere length regulates the epigenetic status of mammalian telomeres and subtelomeres. Nat Genet. 2007; 39: 243-250

52. Ueda Y, Okano M, Williams C, Chen T, Georgopoulos K, Li E. Roles for Dnmt3b in mammalian development: a mouse model for the ICF syndrome. Development. 2006; 133: 1183-1192.

53. Yehezkel S, Segev Y, Viegas-Péquignot E, Skorecki K, Selig S. Hypomethylation of subtelomeric regions in ICF syndrome is associated with abnormally short telomeres and enhanced transcription from telomeric regions. Hum Mol Genet. 2008; 17: 2776-2789.

54. Deng Z, Campbell AE, Lieberman PM. TERRA, CpG methylation and telomere heterochromatin: lessons from ICF syndrome cells. Cell Cycle. 2010; 9: 69-74.

55. Caslini C, Connelly JA, Serna A, Broccoli D, Hess JL. MLL associates with telomeres and regulates telomeric repeat-containing RNA transcription. Mol Cell Biol. 2009; 29: 4519-4526.

56. Arnoult N, Van Beneden A, Decottignies A. Telomere length regulates TERRA levels through increased trimethylation of telomeric H3K9 and HP1a. Nat Struct Mol Biol. 2012; 19: 948-956.

57. De jardin J, Kingston RE. Purification of proteins associated with specific genomic Loci. Cell. 2009; 136: 175-186. 
58. Takahama K, Takada A, Tada S, Shimizu M, Sayama K, Kurokawa R, Oyoshi T. Regulation of telomere length by G-quadruplex telomere DNA- and TERRA-binding protein TLS/FUS. Chem Biol. 2013; 20: 341-350.

59. Porro A, Feuerhahn S, Lingner J. TERRA-reinforced association of LSD1 with MRE11 promotes processing of uncapped telomeres. Cell Rep. 2014; 6: 765-776.

60. Stern JL, Bryan TM. Telomerase recruitment to telomeres. Cytogenet Genome Res. 2008; 122: 243-254.

61. Wang RC, Smogorzewska A, de Lange T. Homologous recombination generates T-loop-sized deletions at human telomeres. Cell. 2004; 119: 355-368.

62. Poulet A, Pisano S, Faivre-Moskalenko C, Pei B, Tauran Y, Haftek-Terreau Z, Brunet F, Le Bihan YV, et al. The N-terminal domains of TRF1 and TRF2 regulate their ability to condense telomeric DNA. Nucleic Acids Res. 2012; 40: 2566-2576.

63. Deng Z, Wang Z, Stong N, Plasschaert R, Moczan A, Chen HS, Hu S. Wikramasinghe P, Davuluri RV, Bartolomei MS, Riethman H, Lieberman PM. A role for CTCF and cohesin in subtelomere chromatin organization, TERRA transcription, and telomere end protection. EMBO J. 2012; 31: 4165-4178.

64. Balk B, Maicher A, Dees M, Klermund J, Luke-Glaser S, Bender K, Luke B. Telomeric RNA-DNA hybrids affect telomere-length dynamics and senescence. Nat Struct Mol Biol. 2013; 20: 1199-1205.

65. LaBranche H, Dupuis S, Ben-David Y, Bani MR, Wellinger RJ, Chabot B. Telomere elongation by hnRNP A1 and a derivative that interacts with telomeric repeats and telomerase. Nat Genet. 1998; 19: 199-202.

66. Zhang QS, Manche L, Xu RM, Krainer AR. hnRNP A1 associates with telomere ends and stimulates telomerase activity. Rna. 2006; 12: 1116-1128.
67. Flynn RL, Centore RC, O'Sullivan RJ, Rai R, Tse A, Songyang Z, Chang S, Karlseder J, Zou L. TERRA and hnRNPA1 orchestrate an RPA-to-POT1 switch on telomeric single-stranded DNA. Nature. 2011; 471: 532-536.

68. Flynn RL, Chang S, Zou L. RPA and POT1: friends or foes at telomeres? Cell Cycle. 2012; 11: 652-657.

69. Le PN, Maranon DG, Altina NH, Battaglia CL, Bailey SM. TERRA, hnRNP A1, and DNA-PKcs Interactions at Human Telomeres. Front Oncol. 2013; 3: 91.

70. Ting NS, Pohorelic B, Yu Y, LeesMiller SP, Beattie TL. The human telomerase RNA component, hTR, activates the DNAdependent protein kinase to phosphorylate heterogeneous nuclear ribonucleoprotein A1. Nucleic Acids Res. 2009; 37: 6105-6115.

71. Zhu X, Kumar R, Mandal M, Sharma N, Sharma HW, Dhingra U, Sokoloski JA, Hsiao R, Narayanan R. Cell cycle-dependent modulation of telomerase activity in tumor cells. Proc Natl Acad Sci USA. 1996; 93: 6091-6095.

72. Reig-Viader R, Vila-Cejudo M, Vitelli V, Buscà R, Sabaté M, Giulotto E, Caldés MG, Ruiz-Herrera A. Telomeric repeat-containing RNA (TERRA) and telomerase are components of telomeres during mammalian gametogenesis. Biol Reprod. 2014; 90: 103.

73. Reig-Viader R, Brieño-Enríquez MA, Khouriauli L, Toran N, Cabero L, Giulotto E, Garcia-Caldés M, Ruiz-Herrera A. Telomeric repeat-containing RNA and telomerase in human fetal oocytes. Hum Reprod. 2013; 28: 414-422.

74. Sampl S, Pramhas S, Stern C, Preusser M, Marosi C, Holzmann K. Expression of telomeres in astrocytoma WHO grade 2 to 4: TERRA level correlates with telomere length, telomerase activity, and advanced clinical grade. Transl Oncol. 2012; 5: 56-65. 